\title{
Interactions of Carvacrol, Caprylic Acid, Habituation, and Mild Heat for Pressure-Based Inactivation of O157 and Non-O157 Serogroups of Shiga Toxin-Producing Escherichia coli in Acidic Environment
}

\author{
Md Niamul Kabir ${ }^{1}$, Sadiye Aras ${ }^{1}$, Abimbola Allison ${ }^{1}$, Jayashan Adhikari ${ }^{1}$, \\ Shahid Chowdhury ${ }^{1}$ and Aliyar Fouladkhah ${ }^{1,2, *}$ \\ 1 Public Health Microbiology Laboratory, Tennessee State University, Nashville, TN 37209, USA; \\ mkabir492@gmail.com (M.N.K.); sadiyearas47@gmail.com (S.A.); abimbolaallison20@gmail.com (A.A.); \\ adkjason99@gmail.com (J.A.); schowdh1@tnstate.edu (S.C.) \\ 2 Cooperative Extension Program, Tennessee State University, Nashville, TN 37209, USA \\ * Correspondence: aliyar.fouladkhah@aya.yale.edu or afouladk@tnstate.edu; Tel.: +1-970-690-7392
}

Received: 2 May 2019; Accepted: 21 May 2019; Published: 23 May 2019 check for
updates

\begin{abstract}
The current study investigated synergism of elevated hydrostatic pressure, habituation, mild heat, and antimicrobials for inactivation of $\mathrm{O} 157$ and non-O157 serogroups of Shiga toxin-producing Escherichia coli. Various times at a pressure intensity level of $450 \mathrm{MPa}$ were investigated at 4 and $45{ }^{\circ} \mathrm{C}$ with and without carvacrol, and caprylic acid before and after three-day aerobic habituation in blueberry juice. Experiments were conducted in three biologically independent repetitions each consist of two replications and were statistically analyzed as a randomized complete block design study using ANOVA followed by Tukey- and Dunnett's-adjusted mean separations. Under the condition of this experiment, habituation of the microbial pathogen played an influential $(p<0.05)$ role on inactivation rate of the pathogen. As an example, $\mathrm{O} 157$ and non-O157 serogroups were reduced $(p<0.05)$ by 1.4 and 1.6 Log CFU/mL after a $450 \mathrm{MPa}$ treatment at $4{ }^{\circ} \mathrm{C}$ for seven min, respectively, before habituation. The corresponding log reductions $(p<0.05)$ after three-day aerobic habituation were: 2.6 , and 3.3 , respectively at $4{ }^{\circ} \mathrm{C}$. Carvacrol and caprylic acid addition both augmented the pressure-based decontamination efficacy. As an example, Escherichia coli O157 were reduced $(p<0.05)$ by 2.6 and $4.2 \log \mathrm{CFU} / \mathrm{mL}$ after a seven-min treatment at $450 \mathrm{MPa}$ without, and with presence of $0.5 \%$ carvacrol, respectively, at $4{ }^{\circ} \mathrm{C}$.
\end{abstract}

Keywords: Shiga toxin-producing Escherichia coli; habituation; carvacrol; caprylic acid; high-pressure pasteurization

\section{Introduction}

The 2015-2020 dietary guidelines of the United States Department of Agriculture recommends an increase in consumption of fruits and vegetables [1]. Over the last two decades, consumption of fresh and processed produce has also been increasing [2]. Contamination of plant-based products prior to consumption is practically unavoidable due to the ubiquitous nature of microbial pathogens and complexity of producing and processing operations $[3,4]$, leading to an array of health and economic complications such as foodborne illnesses, hospitalizations, and death episodes, as well as recalls of food products and foodborne disease outbreaks [4-6].

Contamination with Escherichia coli O157:H7 and non-O157 serogroups of Shiga toxin-producing E. coli are one of the leading concerns of foodborne illnesses linked with muscle- and plant-based 
foods [7-9]. In addition to the Shiga toxin-producing E. coli O157:H7 (STEC) that has historically been linked to an array of food recalls and outbreaks since 1990s [9], non-O157 serogroups of Shiga toxin-producing E. coli (nSTEC) have been gaining increasing public health significance recently due to their emergence in food chain $[9,10]$. The serogroups O26, O45, O103, O111, O121, and O145 (also known as the 'Big Six') are considered as the most epidemiologically significant foodborne serogroups of public health concern among nSTEC [11,12].

Data derived from active surveillance programs of Centers for Disease Control and Prevention [13] indicates that in the United States 3704 and 1579 laboratory confirmed cases occur annually associated with STEC and nSTEC, respectively [13]. It is further estimated that every year in the United States, STEC and nSTEC are responsible for 63,153 and 112,752 domestic foodborne infections, respectively. Among these cases, $68 \%$ of STEC and $82 \%$ of nSTEC cases are foodborne in nature [5]. From 1998 to 2017, at least 590 foodborne outbreaks in the United Sates, including 14 foodborne outbreaks in the state of Tennessee were associated with STEC and/or nSTEC [13].

Although acidification or use of acidic foods are commonly associated with limited multiplication of microorganisms [14], microbial pathogens could survive and proliferate under acidic conditions [15-17]. Particularly, STEC had been involved in several outbreaks of foodborne diseases in different acidic foods, for example: yoghurt [18], mayonnaise [19] and apple cider [20]. It is also observed that acid adaptation can enhance STEC ability to survive in acidic juices for example in asparagus juice $(\mathrm{pH}=3.6)$ and in mango juice ( $\mathrm{pH}=3.2)$ [21]. As an indigenous fruit crop of North America, blueberries have particularly low $\mathrm{pH}$ [22], have been associated with a seven-month STEC outbreak in Massachusetts [13], and thus, could be used as a model for investigating validation studies against STEC and nSTEC in acidic environment.

A viable alternative for pasteurization of products in manufacturing is application of elevated hydrostatic pressure [23]. Unlike traditional thermal processing methods that are typically associated with undesirable physiochemical and organoleptic changes in treated products [24], pressure-based pasteurization could be utilized for assuring safety of the products while minimally affecting their sensory and nutritional composition $[25,26]$. A pressure-based pasteurization could utilizes hydrostatic pressure of 100 to $1000 \mathrm{MPa}$, pressure-intensity level of around $600 \mathrm{MPa}(87 \mathrm{~K} \mathrm{PSI})$ for about three min are currently the most common treatment in the private industry [27]. The main challenge for further adaption of pressure-based pasteurization treatments is slightly higher processing costs associated with the technology, thus, application of pressure treatments at intensity levels below $600 \mathrm{MPa}$, augmented with mild heat and natural antimicrobials could be a desirable approach for the food industry [27].

Caprylic acid is an eight-carbon fatty acid, which could be naturally found in several foods (coconut oil, bovine milk, palm oil, etc.) and is Generally Recognized as Safe by the U.S., Food and Drug Administration as a food additive $[28,29]$. Caprylic acid $\left(\mathrm{C}_{8} \mathrm{H}_{16} \mathrm{O}_{2}\right)$ could be an effective antimicrobial compound against Gram-negative and Gram-positive foodborne pathogens such as E. coli O157: H7, Listeria monocytogenes and Salmonella serovars [28,30-32]. Carvacrol $\left(\mathrm{C}_{10} \mathrm{H}_{14} \mathrm{O}\right)$, found primarily in oregano, is another natural bioactive compound with reported antimicrobial properties [33] and is broadly known for its effective antioxidant and antimicrobial activity [34,35].

The purpose of this study was to investigate the role of mild heat and addition of caprylic acid and carvacrol on decontamination efficacy of a pressure-based pasteurization treatment against STEC and nSTEC. Habituation of the pathogen, as further delineated in Section 2.1, in an acidic food vehicle were also investigated as an important element for maximizing external validity of a decontamination hurdle validation study.

\section{Materials and Methods}

\subsection{Escherichia coli Strains, Preparation of Culture, Habituation, and Inoculation}

A six-strain mixture of Shiga toxin-producing E. coli O157:H7 (STEC) (ATCC ${ }^{\circledR}$, Manassas, VA, USA, numbers BAA 460, 43888, 43894, 35150, 43889 and 43890) and a six-strain mixture of 'Big 
Six' non-O157 Shiga toxin-producing E. coli (nSTEC) strains, including O26:H11, O45:H2, O103:H2, O111:NM, O121:H19, and O145 (ATCC ${ }^{\circledR}$ numbers BAA 2196, BAA 2193, BAA 2215, BAA 2440, BAA 2219 and BAA 2192 respectively) were used in this study for inoculation of sterilized (autoclaved at $121^{\circ} \mathrm{C}$, for $15 \mathrm{~min}$, under 15 PSI) blueberry juice. The STEC and nSTEC strains with public health significance and those derived from our previously published strain selection trials were selected for this study [9].

The cultures for each of the above-mentioned strains, obtained from American Type Culture Collection (Manassas, VA, USA), were grown on Tryptic Soy Agar (Difco, Becton Dickinson, Franklin Lakes, NJ, USA) supplemented with $0.6 \%$ yeast extract (TSA + YE) and for $24 \mathrm{~h}$ incubated at $37^{\circ} \mathrm{C}$. Forty eight hours before each experiment, a loopful of single colony of each STEC or nSTEC strains was aseptically transferred for activation into $10 \mathrm{~mL}$ Tryptic Soy Broth (Difco, Becton Dickinson, Franklin Lakes, NJ, USA) supplemented with $0.6 \%$ yeast extract (TSB + YE). Use of this media and the supplement minimizes acid stress of the bacterial cells during incubation at $37^{\circ} \mathrm{C}$ for $20-24 \mathrm{~h}[23,27,36]$. After incubation for $20-24 \mathrm{~h}$ at $37^{\circ} \mathrm{C}, 100-\mu \mathrm{L}$ aliquot of the culture was individually and aseptically sub-cultured into another $10 \mathrm{~mL}$ of TSB $+\mathrm{YE}$, for $22-24 \mathrm{~h}$ at $37^{\circ} \mathrm{C}$, for each of the 12 strains, separately.

Each overnight sub-cultured strain ( $2 \mathrm{~mL}$ per strain) was then harvested by centrifugation (Model 5424, Eppendorf North America, Hauppauge, NY, USA; Rotor FA-45-24-11) at 6000 RPM (3548 $\mathrm{g}$ for $88 \mathrm{~mm}$ rotor) for $15 \mathrm{~min}$. Bacterial pellets were then re-suspended in $2 \mathrm{~mL}$ Phosphate Buffered Saline (VWR International, Radnor, PA, USA) and washed twice by centrifugation with the above-mentioned intensity and time to remove growth media, excreted secondary metabolites, and sloughed cell components. Two separate six-strain bacterial cocktails (for STEC and nSTEC) were made by combining the washed and re-suspended strains into PBS (VWR International, Radnor, PA, USA), and were used as the inocula for this study. Non-habituated samples were prepared by 10-fold dilution of each of the STEC and nSTEC cocktails in PBS followed by inoculating sterilized blueberry juice samples for target population of 5-6 Log CFU/mL. The habituated samples were prepared by adding $10 \mathrm{~mL}$ of STEC and nSTEC cocktails (separately for each strain mixture) to $40 \mathrm{~mL}$ of sterilized blueberry juice, followed by a $72 \mathrm{~h}$ aerobic storage at $4{ }^{\circ} \mathrm{C}$ [23]. Habituation allows pathogen acclimatization to intrinsic factor and temperature of the food product and could impact external validity of a microbial challenge study [37-39]. Levels of inoculation for habituated and non-habituated samples and below-mentioned temperatures and concentrations of antimicrobials were selected after conduct of preliminary trials.

\subsection{Preparation of Antimicrobials, and Mild Heat and Pressure-Based Pasteurization}

Two naturally occurring antimicrobial compounds (carvacrol and caprylic acid) were used in this study for inactivation of 72-h habituated STEC and nSTEC in sterilized blueberry juice at two temperatures and at an elevated hydrostatic pressure level of $450 \mathrm{MPa}$. The temperature of the trials were precisely controlled using a water jacket surrounding the treatment chamber, connected to a circulating water bath and monitored by k-type thermocouples as delineated in details in our recent open access publications [23,27]. For $4{ }^{\circ} \mathrm{C}$ experiments, $0.5 \%(7.5 \mu \mathrm{L}$ of antimicrobial in $1.5 \mathrm{~mL}$ of inoculated product $(v / v))$ and for $45^{\circ} \mathrm{C}$ experiment, $0.1 \%$ concentration $(1.5 \mu \mathrm{L}$ of antimicrobial in $1.5 \mathrm{~mL}$ of inoculated product $(v / v))$ of carvacrol and caprylic acid were used based on the above-mentioned preliminary trials. In each experiment, the concentration of antimicrobials was prepared aseptically in sterilized blueberry juice. Inoculated blueberry juice were then exposed to 450 Megapascal (MPa), i.e., c. 65,000 pounds per square inch (PSI) hydrostatic pressure (Barocycler Hub880 Explorer, Pressure Bioscience Inc., South Easton, MA, USA) at 4 and $45^{\circ} \mathrm{C}$ for the time intervals of 0 (untreated control) to $7 \mathrm{~min}$. Samples containing antimicrobials were also tested immediately after addition of the antimicrobial and prior to pressure treatment (treated control). The treatments were carried out in no-disk PULSE (Pressure BioScience Inc., South Easton, MA, USA) containing $1.5 \mathrm{~mL}$ of inoculated blueberry juice. The PULSE tubes were then used for hydrostatic pressure treatment with 1,3,5 and 7 min holding time, in addition to the above-mentioned controls. Pressure and temperature of trials 
were monitored and recorded automatically every 3 s using HUB Explorer PBI (Version 1.0.8, Pressure BioScience Inc., South Easton, MA, USA) software.

\subsection{The $p H$, Neutralization, and Microbiological Analyses}

Each treated sample was neutralized using $5 \mathrm{~mL}$ of D/E neutralizing broth (Difco, Becton Dickinson, Franklin Lakes, NJ, USA) to reduce the effect of food vehicle's intrinsic factors before microbiological analyses. The detection limit of microbiological analyses was, thus, $0.48 \log \mathrm{CFU} / \mathrm{mL}$. After neutralization, to enhance the recovery of injured cells, samples were 10-fold serially diluted in Maximum Recovery Diluent (Difco, Becton Dickinson, Franklin Lakes, NJ, USA) and then plated on TSA media supplemented with $0.6 \%$ yeast extract (TSA + YE). All plates were incubated for $24-48 \mathrm{~h}$ at $37^{\circ} \mathrm{C}$. After incubation, colony forming units were counted manually and converted into log values for further statistical analyses. The $\mathrm{pH}$ of treated samples was measured two times (after treatment and before neutralization, as well as after neutralization) using a digital $\mathrm{pH}$ meter (Mettler Toledo AG, Grelfensee, Switzerland) calibrated at $\mathrm{pH}$ levels of 4, 7 and 10 before measurements.

\subsection{Statistical Analyses and Experimental Design}

The sample size of this study was determined to be at least 5 repetitions per treatment to achieve statistical power of $80 \%$. This sample size was obtained from a previous a priori power analysis using Proc Power of SAS software (version 9.2, SAS Institute, Cary, NC, USA) using existing pressure-treated products in the public health microbiology laboratory [40]. The present study was conducted at two temperatures of 4 and $45^{\circ} \mathrm{C}$ using two inocula of STEC and nSTEC. At each temperature, the study contained three biologically independent repetitions (three blocks), each consisted of 2 replications. Each replication was also microbiologically analyzed in duplicate (microbiological replications). Thus each reported value is a mean of 12 individual analyses (i.e., 3 blocks, 2 replications, and 2 microbiological repetitions). Initial data arrangement, $\log$ transformations and descriptive analysis of the data were completed using Microsoft Excel. The study was considered as a randomized complete block design, and log-transformed microbial counts were statistically analyzed using generalized liner model of SAS for conduct of ANOVA followed by Tukey- and Dunnett's-adjusted mean separations at type I error level of $5 \%(a l p h a=0.05)$. In order to calculate inactivation indices (D-value and $\mathrm{K}_{\max }$ ) Microsoft Excel and GInaFiT (version 1.7, Katholieke Universiteit, Leuven, Belgium) [41] software were used, respectively.

\section{Results and Discussion}

As previously delineated in Section 2.2, the experiments were conducted under controlled temperatures to assure microbial inactivation could be attributed to the intrinsic and extrinsic factors of interest rather than temperature fluctuations. Samples treated at 4 and $45^{\circ} \mathrm{C}$, had similar $(p \geq 0.05)$ temperature values (mean $\pm \mathrm{SD}$ ) before and after the treatments. Across all treatments at $4{ }^{\circ} \mathrm{C}$, the values before treatments were $4.8 \pm 0.2^{\circ} \mathrm{C}$ and were $4.9 \pm 0.2{ }^{\circ} \mathrm{C}$ after the treatments. Values were ranging from 4.3 to $5.2{ }^{\circ} \mathrm{C}$ and 4.3 to $5.3^{\circ} \mathrm{C}$, before and after treatments, respectively. For samples treated at $45^{\circ} \mathrm{C}$ as well, temperature recordings were similar $(p<0.05)$ before and after treatments. The temperature values were $44.5 \pm 0.3$ and $44.8 \pm 0.4{ }^{\circ} \mathrm{C}$, before and after treatments, respectively. The range for the recordings were from 43.7 to $45.0^{\circ} \mathrm{C}$ and 43.7 to $45.2^{\circ} \mathrm{C}$ for samples prior and after treatments, respectively. Extent of precision in control of temperature could be further delineated through calculation of coefficient of variation (CV) associated with the temperature recordings. The CVs associated with $4{ }^{\circ} \mathrm{C}$ samples were $4.51 \%$ and $4.57 \%$ and for samples treated at $45{ }^{\circ} \mathrm{C}$ were $0.58 \%$ and $0.76 \%$, before and after treatments, respectively.

The $\mathrm{pH}$ levels of the samples were also similar $(p \geq 0.05)$ before and after treatments. For samples treated at $4{ }^{\circ} \mathrm{C}$, and prior to neutralization, the $\mathrm{pH}$ value (mean $\pm \mathrm{SD}$ ) and range were $3.16 \pm 0.0$ and 3.12 to 3.22 , respectively. After neutralization, these values were expectedly increased $(p<0.05)$ to $5.56 \pm 0.27$, ranging from 5.25 to 6.02 . Similarly, for samples treated at $45{ }^{\circ} \mathrm{C}$, these values were 
$3.33 \pm 0.1$ and $5.54 \pm 0.1$, before and after neutralization. These values were ranging from 3.24 to 3.44 and 5.37 to 5.69 before and after neutralization, respectively. The $\mathrm{CVs}$ associated with $\mathrm{pH}$ measurements were $0.42 \%$ ( $\left({ }^{\circ} \mathrm{C}\right.$ samples, without neutralization), $4.89 \%$ (neutralized $4{ }^{\circ} \mathrm{C}$ samples), $1.61 \%\left(45^{\circ} \mathrm{C}\right.$ samples, without neutralization), and $1.70 \%$ (neutralized, $45^{\circ} \mathrm{C}$ samples).

\subsection{Pressure-Based Pasteurization of O157 and Non-O157 Serogroups of Shiga Toxin-Producing Escherichia} coli at $4^{\circ} \mathrm{C}$, Before and After Habituation

As further delineated in Section 2.1, this study utilized two separate inoculated products for the pressure-based microbial challenge studies using a six-strain mixture of O157 Shiga toxin-producing Escherichia coli (STEC) and a six-strain non-O157 mixture of O26, O45, O103, O111, O121, and O145 Shiga toxin-producing Escherichia coli (nSTEC). Data associated with the current study is also provided as a supplementary file. At $4{ }^{\circ} \mathrm{C}$ and after the habituation, the STEC and nSTEC counts (mean \pm SD) of blueberry juice were $6.32 \pm 0.5$ and $6.12 \pm 0.6 \mathrm{Log} C F U / \mathrm{mL}$, respectively (Figure 1A). Hydrostatic pressure treatment of $450 \mathrm{MPa}$ (c. $65 \mathrm{~K} \mathrm{PSI}$ ), for 1, 3, 5, and $7 \mathrm{~min}$, reduced the STEC by 1.7 to 2.6 $\log \mathrm{CFU} / \mathrm{mL}$ and specifically reduced $(p<0.05)$ the STEC counts to $4.60 \pm 0.8,4.45 \pm 0.9,4.51 \pm 0.8$, $3.68 \pm 1.1$, respectively (Figure 1A). Sensitivity of nSTEC were similar to STEC- the treatments for 1 , 3,5 , and $7 \mathrm{~min}$ at the above-referenced pressure and intensity level lead to 1.1, 2.7, 2.6, and $3.3 \mathrm{log}$ reductions of nSTEC samples (Figure 1A). Under the condition of our experiment, habituation played an influential role on sensitivity of both STEC and nSTEC serogroups to pressure-based treatments at $4{ }^{\circ} \mathrm{C}$ (Figure 1A,B). For non-habituated samples at $4{ }^{\circ} \mathrm{C}$, STEC and nSTEC counts were $5.55 \pm 0.6$ and $5.00 \pm 0.1$ prior to treatments, respectively. The STEC were reduced $(p<0.05)$ to $4.35 \pm 0.3,4.26 \pm 0.5$, $4.57 \pm 0.9$, and $4.13 \pm 0.2 \log \mathrm{CFU} / \mathrm{mL}$, after treatments for $1,3,5$, and $7 \mathrm{~min}$ at $450 \mathrm{MPa}$, respectively (Figure 1B). These reductions were considerably less that reductions of the habituated STEC. In other words, the habituated STEC were more sensitive to pressure-based treatments at this temperature relative to the non-habituated phenotype. As an example, $7 \mathrm{~min}$ of treatment at $450 \mathrm{MPa}$ at $4{ }^{\circ} \mathrm{C}$ reduced the habituation STEC $(p<0.05)$ by $3.7 \log \mathrm{CFU} / \mathrm{mL}$ (Figure $1 \mathrm{~A}$ ), while the same treatment were only capable of reducing $(p<0.05)$ the non-habituated STEC for $1.4 \log \mathrm{CFU} / \mathrm{mL}$ (Figure 1B). This trend was also observed for habituated and non-habituated nSTEC (Figure 1A,B).

This considerable difference in sensitivity of the pathogen before and after habituation had been discussed in the microbiology literature in the past. While studies, similar to our current study, had observed that post-stress, pathogens exhibit more sensitivity to a decontamination treatment. Some studies also indicate certain stressors could lead to cross-protective effects, i.e., increasing the tolerance of a pathogen post-stress $[23,38,42,43]$. If a manufacturer is relying on validation studies with non-habituated inoculated pathogen, the validation data could be an overestimation or underestimation of the treatment decontamination efficacy, and thus, leading to false sense of treatment efficacy or a treatment that is overly conservative. This could also lead to over- or under-estimation of microbial reductions in risk assessment analyses throughout the supply chain. It is thus recommended that habituation for each specific product-pathogen-treatment combination be considered as an important factor of a validation study to assure data obtained from a microbial challenge study has external validity and is conducted in an environment that is as close as possible to actual processing condition of a product. This could assure economic feasibility of a treatment as well as providing assurance that a treatment is safeguarding the public health. Currently, there is a knowledge gap about sensitivity of acid-adapted and acid-stressed foodborne pathogens of public health concern to various pressure-based treatments relative to their wild-type phenotypes. 

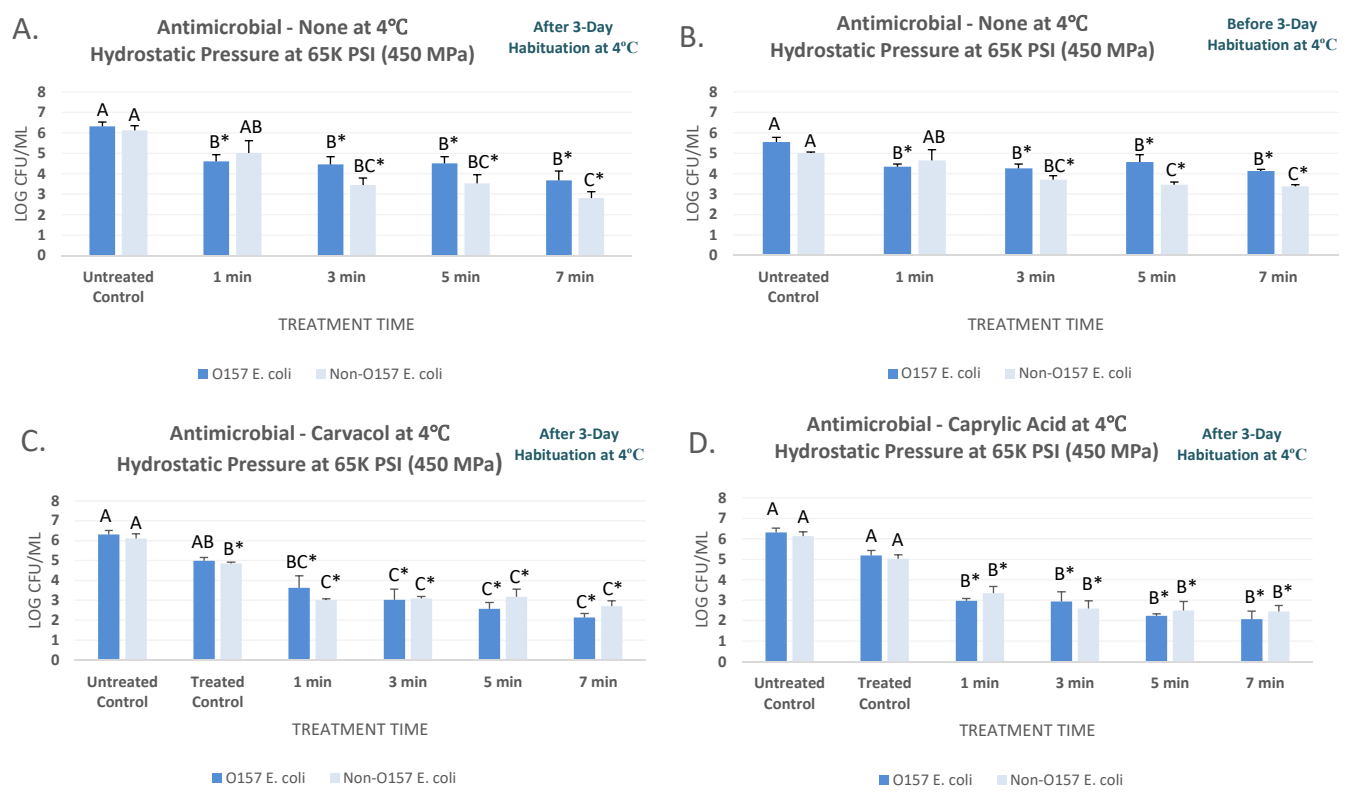

Figure 1. Inactivation of six-strain cocktail of habituated and non-habituated E. coli O157:H7 (ATCC ${ }^{\circledR}$ numbers BAA 460, 43888, 43894, 35150, 43889, 43890) and the 'Big Six' non-O157 E. coli mixtures (ATCC ${ }^{\circledR}$ numbers BAA 2196, BAA 2193, BAA 2215, BAA 2440, BAA 2219, BAA 2192) in sterilized blueberry juice, treated by carvacrol $(0.5 \%)$, caprylic acid $(0.5 \%)$ and elevated hydrostatic pressure at $450 \mathrm{MPa}$ (Barocycler Hub880 Explorer, Pressure Bioscience Inc., South Easton, MA, USA) for 0, 1, 3,5 , and $7 \mathrm{~min}$ at $4{ }^{\circ} \mathrm{C}$. In each graph, and for each pathogen mixture separately, columns of each time interval followed by different uppercase letters are representing $\log \mathrm{CFU} / \mathrm{mL}$ values (Mean $\pm \mathrm{SE}$ ) that are statistically $(p<0.05)$ different (Tukey-adjusted ANOVA). Uppercase letters followed by * sign are statistically $(p<0.05)$ different than the untreated control (not treated with antimicrobial) (Dunnett's-adjusted ANOVA). (A) After 3 days of habituation, treated by no antimicrobial at $4{ }^{\circ} \mathrm{C}$; (B) Before 3 days of habituation, treated by no antimicrobial at $4{ }^{\circ} \mathrm{C}$; (C) After 3 days of habituation, treated by $0.5 \%$ carvacrol at $4{ }^{\circ} \mathrm{C}$; (D) After 3 days of habituation, treated by $0.5 \%$ caprylic acid at $4{ }^{\circ} \mathrm{C}$.

\subsection{Augmenting the Efficacy of High Pressure Pasteurization using Carvacrol and Caprylic Acid at $4{ }^{\circ} \mathrm{C}$}

Under the condition of our experiments, we observed the selected two natural antimicrobials could appreciably augment the efficacy of the pressure-based pasteurization of STEC and nSTEC at $4{ }^{\circ} \mathrm{C}$. It is noteworthy that the synergism of elevated hydrostatic pressure and carvacrol and caprylic acid were investigated on inoculated samples with three-day aerobic habituation that, as discussed in Section 3.1, yields more realistic outcome with higher external validity. Data and graphical representations obtained and reported for these experiments were similar in structure to those elaborated in Section 3.1 with the exception that the microbial reductions immediately after exposure to $0.5 \%$ antimicrobial were also determined, thus graphs contain untreated controls as well as treated controls (e.g., samples that are immediately neutralized and enumerated after exposure to the antimicrobial).

The STEC and nSTEC counts (mean \pm SD) for untreated controls were $6.32 \pm 0.5$ and $6.12 \pm 0.6$ $\log \mathrm{CFU} / \mathrm{mL}$, respectively at $4{ }^{\circ} \mathrm{C}$. Immediately after exposure to $0.5 \%$ carvacrol, these counts were reduced $(p<0.05)$ to $4.99 \pm 0.4$ and $4.86 \pm 0.1 \log$ CFU/mL, for STEC and nSTEC samples, respectively (Figure 1C). Carvacrol were able to enhance $(p<0.05)$ the efficacy of the treatment. As an example, treatments of STEC samples for 5 and $7 \mathrm{~min}$ at $450 \mathrm{MPa}$ at $4{ }^{\circ} \mathrm{C}$ lead to 3.8 and $4.2 \log \mathrm{CFU} / \mathrm{mL}$ reductions $(p<0.05)$ while same treatment at the same temperature and intensity level without presence of carvacrol resulted in 1.0 and $1.4 \log \mathrm{CFU} / \mathrm{mL}$ reductions $(p<0.05)$ in habituated samples, respectively (Figure 1A,C). In vast majority of tested time intervals, STEC and nSTEC serogroups exhibited comparable sensitivity to high hydrostatic pressure (Figure 1A-D). Caprylic acid, at $0.5 \%$ concentration, were similarly effective to augment the decontamination efficacy of the pressure-based treatments at $4{ }^{\circ} \mathrm{C}$. The nSTEC counts, as an example, were $6.12 \pm 0.6 \log \mathrm{CFU} / \mathrm{mL}$ prior to treatment 
and prior to exposure to caprylic acid (untreated control). These counts were reduced $(p<0.05)$ to $5.02 \pm 0.5 \log \mathrm{CFU} / \mathrm{mL}$ immediately after exposure to $0.5 \%$ caprylic acid (treated controls) and were further reduced $(p<0.05)$ to $3.35 \pm 0.8,2.60 \pm 0.9,2.49 \pm 1.1,2.44 \pm 0.8 \log \mathrm{CFU} / \mathrm{mL}$ after 1-, 3-, 5-, and 7-min treatments at $450 \mathrm{MPa}$ at $4{ }^{\circ} \mathrm{C}$ (Figure 1D). These reductions were appreciably higher than those obtained from elevated hydrostatic pressure alone for both STEC and nSTEC. As an example, the above-reference 7-min treatment reduced $(p<0.05)$ the STEC and nSTEC for 4.2 and $3.7 \log$ CFU $/ \mathrm{mL}$ in presence of $0.5 \%$ caprylic acid, respectively, while the same treatment resulted in 1.4 and $1.6 \mathrm{log}$ $\mathrm{CFU} / \mathrm{mL}$ reductions $(p<0.05)$ for the habituated samples without caprylic acid (Figure $1 \mathrm{~A}, \mathrm{D})$.

These results could be of practical importance for the private industry with a high-pressure processing plant. At current times, slightly higher operation costs of many pressure-treated products relative to existing heat-treated commodities in the market are the main curtailment for further expanding the utilization of this technology in the food processing industry [23,27]. Main costs of the operation are associated with maintenance and energy expenditure associated with use of high levels of hydrostatic pressure. Our study indicates that lower levels of pressure could lead to similar decontamination efficacy in presence of natural antimicrobials such as carvacrol and caprylic acid.

\subsection{Pressure-Based Pasteurization of the Pathogen at $45^{\circ} \mathrm{C}$ as Affected by Habituation, Carvacrol and Caprylic Acid}

The pressure treatments discussed in Sections 3.1 and 3.2, coupled with mild heat were appreciably more efficacious for decontamination of the product from STEC and nSTEC (Figure 2A-C). This thermal-assisted pressure-based treatment at $450 \mathrm{MPa}$ and $45^{\circ} \mathrm{C}$ were able to reduce $(p<0.05)$ the STEC counts by 3.8, 4.0, 4.8, and $5.4 \log \mathrm{CFU} / \mathrm{mL}$ for habituated samples (Figure 2A). This decontamination efficacy were also observed with similar trends for the nSTEC samples, leading to 3.3 to $4.8 \mathrm{log}$ $\mathrm{CFU} / \mathrm{mL}$ reductions for treatments of up to $7 \mathrm{~min}$ (Figure 2A). Effects of habituation at this temperature were less pronounced relative to the experiment conducted at $4{ }^{\circ} \mathrm{C}$ (Figure 2A). As an example, counts of non-habituated STEC and nSTEC samples were $5.96 \pm 0.3$ and $5.88 \pm 0.5$ before treatments and were reduced $(p<0.05)$ to $0.66 \pm 0.2$ and $0.91 \pm 0.7 \log \mathrm{CFU} / \mathrm{mL}$ after 7 -min treatments at $450 \mathrm{MPa}$ and $45^{\circ} \mathrm{C}$, respectively. Counts for habituated STEC and nSTEC were reduced $(p<0.05)$ by 5.4 and $4.8 \log$ values, similar to the reductions obtained by treatment of non-habituated samples (Figure 2A,B). Our data indicates, habituation could have a more pronounced effect on external validity of a pressure-based validation study at $4{ }^{\circ} \mathrm{C}$ while may have only modest effects on validity of a thermal-assisted high-pressure processing.

At elevated temperature, effects of carvacrol and caprylic acid at $0.1 \%$ were also less pronounced in augmenting the decontamination efficacy of the treatments (Figure 2C,D). This indicates that while these antimicrobials might be efficacious alone, or coupled with pressure-based treatments at lower temperature, at $0.5 \%$ concentrations, but these do not augment the efficacy of a treatment at higher temperature when tested at $0.1 \%$. Similar effects were observed in the past when acidic acid was not able to augment efficacy of a heat treatment at elevated temperature while efficacious at ambient environment [44]. As an example, STEC counts of habituated samples treated without antimicrobial, with $0.1 \%$ carvacrol, and with $0.1 \%$ caprylic acid for $3 \mathrm{~min}$ at $450 \mathrm{MPa}$ were similar $(p \geq 0.05)$ and were $3.09 \pm 1.3,3.91 \pm 0.4$, and $3.79 \pm 0.6 \log \mathrm{CFU} / \mathrm{mL}$, respectively (Figure 2A,C,D). Similar to treatments at lower temperature, STEC and nSTEC counts were comparable for the vast majority of time and pressure treatments, prior and after habituation, and in presence or absence of the antimicrobials (Figure 2A-D). Our results, thus indicate that mild elevated heat and natural antimicrobial could augment efficacy of a pressure-based pasteurization with similar effectiveness against STEC and nSTEC, but utilization of both mild heat and antimicrobials simultaneously does not necessarily provide added decontamination benefit. 

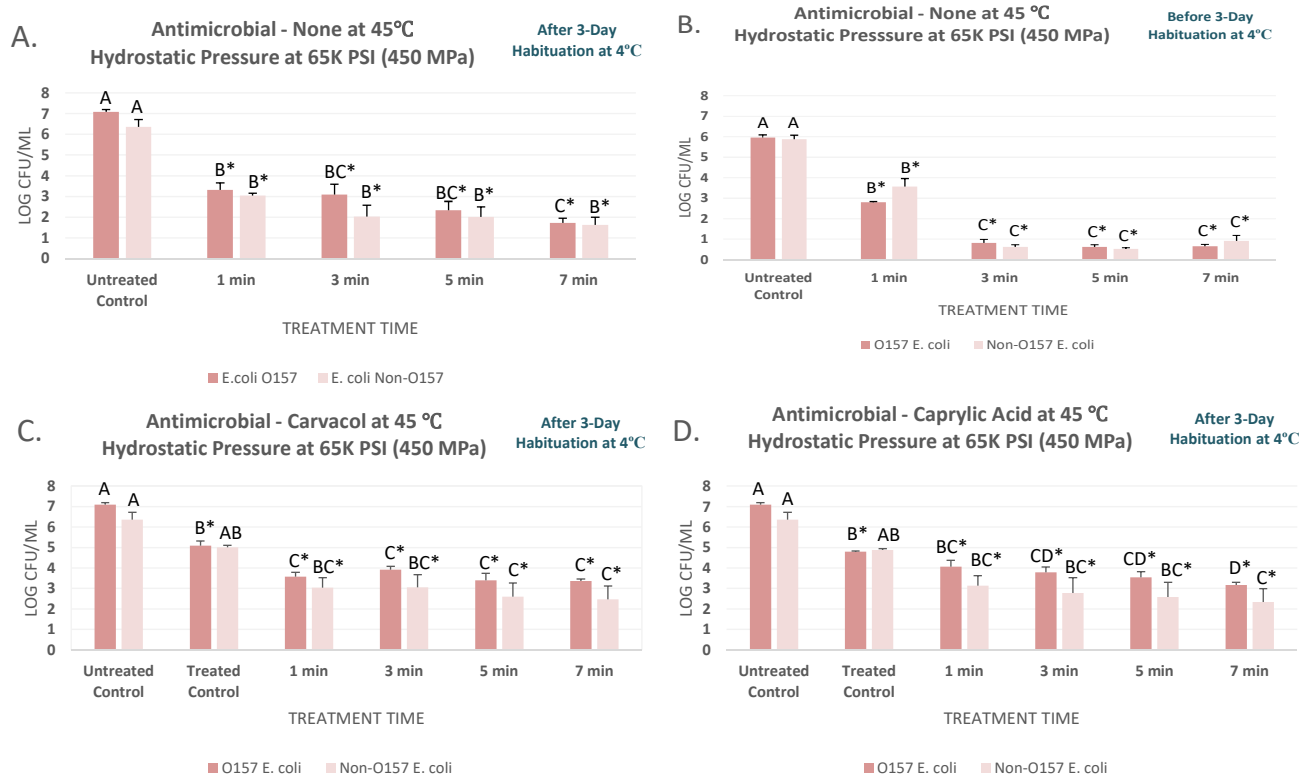

Figure 2. Inactivation of six-strain cocktail of habituated and non-habituated E. coli O157:H7 (ATCC ${ }^{\circledR}$ numbers BAA 460, 43888, 43894, 35150, 43889, 43890) and the 'Big Six' non-O157 E. coli strain mixtures (ATCC ${ }^{\circledR}$ numbers BAA 2196, BAA 2193, BAA 2215, BAA 2440, BAA 2219, BAA 2192) in sterilized blueberry juice, treated by carvacrol $(0.1 \%)$, caprylic acid $(0.1 \%)$ and elevated hydrostatic pressure at $450 \mathrm{MPa}$ (Barocycler Hub880 Explorer, Pressure Bioscience Inc., South Easton, MA, USA) for 0, 1, 3,5 , and $7 \mathrm{~min}$ at $45^{\circ} \mathrm{C}$. In each graph, and for each pathogen mixture separately, columns of each time interval followed by different uppercase letters are representing $\log \mathrm{CFU} / \mathrm{mL}$ values (mean $\pm \mathrm{SE}$ ) that are statistically $(p<0.05)$ different (Tukey-adjusted ANOVA). Uppercase letters followed by * sign are statistically $(p<0.05)$ different than the untreated control (not treated with antimicrobial) (Dunnett's-adjusted ANOVA). (A) After three days of habituation, treated by no antimicrobial at $45{ }^{\circ} \mathrm{C}$; (B) Before three days of habituation, treated by no antimicrobial at $45{ }^{\circ} \mathrm{C}$; (C) After three days of habituation, treated by $0.1 \%$ carvacrol at $45^{\circ} \mathrm{C}$; (D) After three days of habituation, treated by $0.1 \%$ caprylic acid at $45^{\circ} \mathrm{C}$.

3.4. Linear and Non-Leaner Inactivation Indices for High Pressure Pasteurization of O157 and Non-O157 Serogroups of Shiga Toxin-Producing Escherichia coli at 4 and $45^{\circ} \mathrm{C}$

Effects of habituation and synergism of heat, carvacrol and/or caprylic acid with the pressure-based pasteurization could be further discussed by interpretation of linear and non-linear inactivation indices (Figures 3 and 4). D-value was the linear model utilized in this study that could be interpreted as the time required at the specific condition of the experiment to achieve $90 \%$ reduction of the inoculated pathogen (i.e., one-log reduction). A non-linear model had also been utilized in this study using GlnaFiT version 1.7 software [41]. The reported $k_{\max }$ values are in unit of $1 /$ min thus smaller $K_{\max }$ values indicate longer time required for reduction of the pathogen, in contrast to D-value that is in unit of $\min$.

The D-value for STEC for habituated and non-habituated samples (Figure 3A,C) emphasizes on importance of this practice on outcome of a challenge study. The D-value associated with habituated STEC were $13.70 \mathrm{~min}$ while for non-habituated samples this inactivation index was 7.76 min (Figure 3A,C). This effect was not observed at higher temperature. At $45^{\circ} \mathrm{C}$, the D-values were similar for habituated and non-habituated STEC samples and were 1.65 and $1.51 \mathrm{~min}$, respectively (Figure 3A,C).

Carvacrol was able to augment the efficacy of pressure-based pasteurization of the pathogen as evidenced by inactivation indices. As an example, nSTEC required $8.03 \mathrm{~min}$ of treatment at $450 \mathrm{MPa}$ and $4{ }^{\circ} \mathrm{C}$ for one-log reduction e.g., D-value $=8.03 \mathrm{~min}$ (Figure $3 \mathrm{~B}$ ). In presence of $0.5 \%$ carvacrol, same treatment required only $2.92 \mathrm{~min}$ for one-log reduction (Figure $3 \mathrm{~F}$ ). The $\mathrm{k}_{\max }$ values also delivered similar trend, having values of 2.77 and 13.19 1/min for nSTEC samples without carvacrol, and those 
treated with presence of $0.5 \%$ carvacrol (Figure $3 \mathrm{~B}, \mathrm{~F}$ ). At $4{ }^{\circ} \mathrm{C}, 0.5 \%$ caprylic acid was also capable of reducing the time for one-log reduction of both STEC and nSTEC as exhibited in Figure 3A,B,G,H.

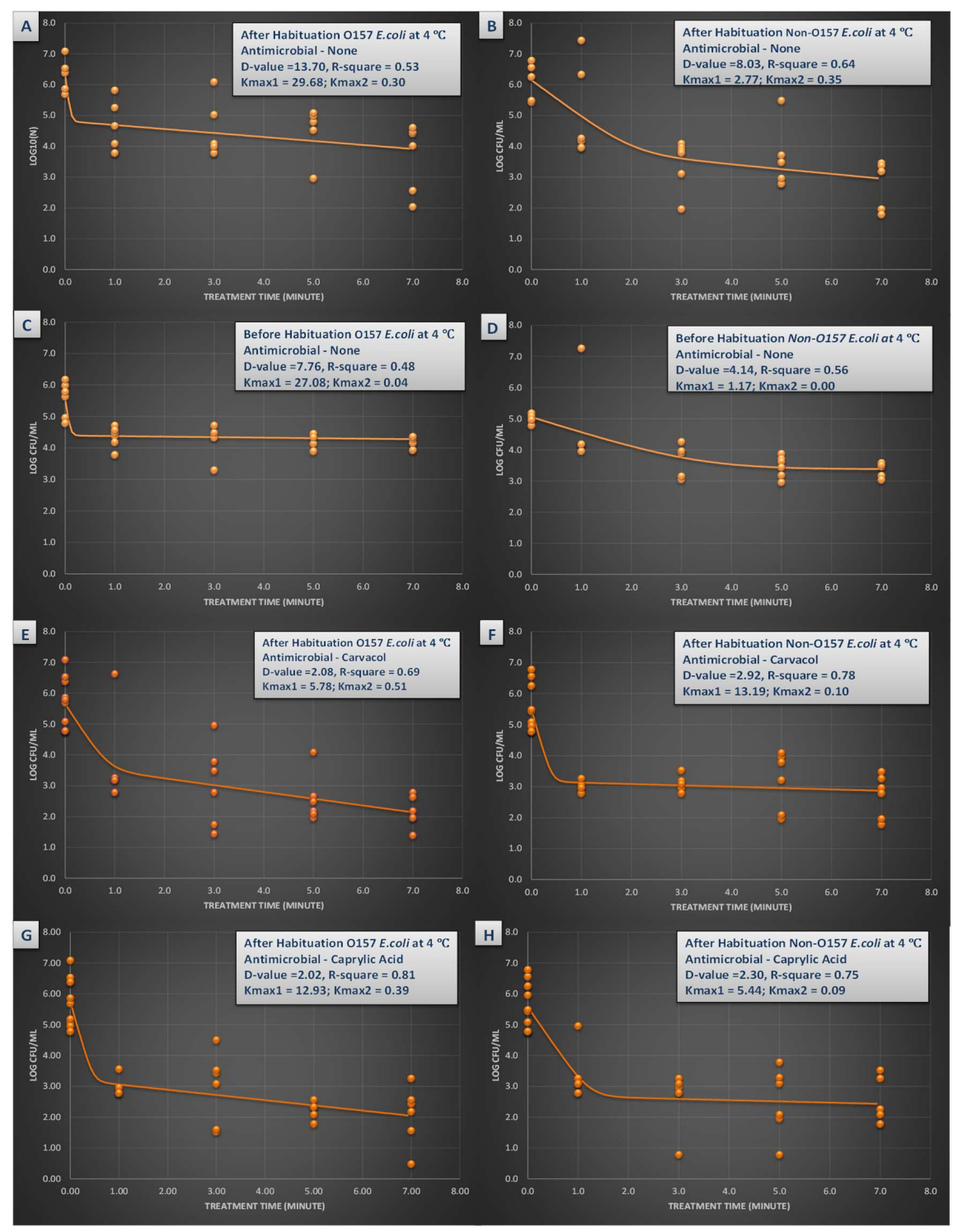

Figure 3. Inactivation rates for six-strain habituated and non-habituated mixture of E. coli O157:H7 (ATCC ${ }^{\circledR}$ numbers BAA 460, 43888, 43894, 35150, 43889, 43890) and the 'Big Six' non-O157 E. coli strain mixtures (ATCC ${ }^{\circledR}$ numbers BAA 2196, BAA 2193, BAA 2215, BAA 2440, BAA 2219, BAA 2192) exposed to $0.5 \%$ carvacrol, $0.5 \%$ caprylic acid, and elevated hydrostatic pressure at $450 \mathrm{MPa}$ (Barocycler Hub 440, Pressure BioScience Inc., South Easton, MA) in sterilized blueberry juice at $4{ }^{\circ} \mathrm{C}$. Using the GInaFiT software, the provided $\mathrm{K}_{\max }$ values are selected from the best-fitted model (goodness-of-fit indicator of $R^{2}$ values, $\left.\alpha=0.05\right)$. $K_{\max }$ values indicate the expressions of number of log cycles of reduction in $1 /$ min unit for each pressure/temperature combinations. Presented D-values are calculated based on best-fitted linear model, showing time required for one $\log (90 \%)$ of microbial cell reductions of the microbial cell mixture. (A) Habituated E. coli $\mathrm{O} 157$ treated by no antimicrobial at $4{ }^{\circ} \mathrm{C}$ with $\mathrm{R}^{2}=0.53$; (B) Habituated E. coli non-O157 treated by no antimicrobial at $4{ }^{\circ} \mathrm{C}$ with $\mathrm{R}^{2}=0.64$; (C) Non-habituated E. coli $\mathrm{O} 157$ treated by no antimicrobial at $4{ }^{\circ} \mathrm{C}$ with $\mathrm{R}^{2}=0.48$; (D) Non-habituated E. coli non-O157 treated by no antimicrobial at $4{ }^{\circ} \mathrm{C}$ with $\mathrm{R}^{2}=0.56$; (E) Habituated $E$. coli $\mathrm{O} 157$ treated by carvacrol $(0.5 \%)$ at $4{ }^{\circ} \mathrm{C}$ with $\mathrm{R}^{2}=0.69 ;(\mathrm{F})$ Habituated E. coli non-O157 treated by carvacrol $(0.5 \%)$ at $4{ }^{\circ} \mathrm{C}$ with $\mathrm{R}^{2}=0.78$; (G). Habituated E. coli $\mathrm{O} 157$ treated by caprylic acid $(0.5 \%)$ at $4{ }^{\circ} \mathrm{C}$ with $\mathrm{R}^{2}=0.81 ;(\mathbf{H})$. Habituated $E$. coli non-O157 treated by caprylic acid $(0.5 \%)$ at $4{ }^{\circ} \mathrm{C}$ with $\mathrm{R}^{2}=0.75$. 

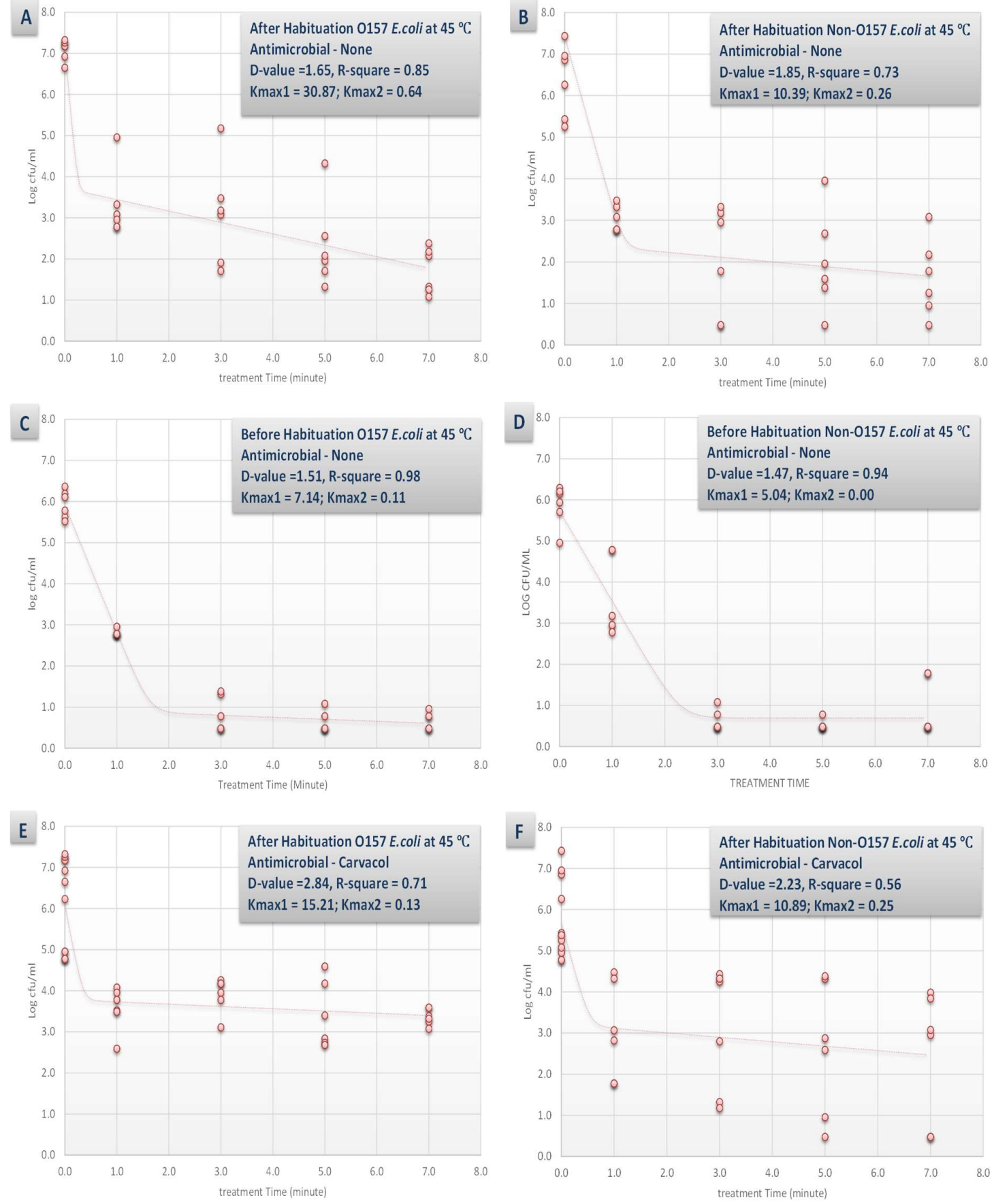

Figure 4. Cont. 

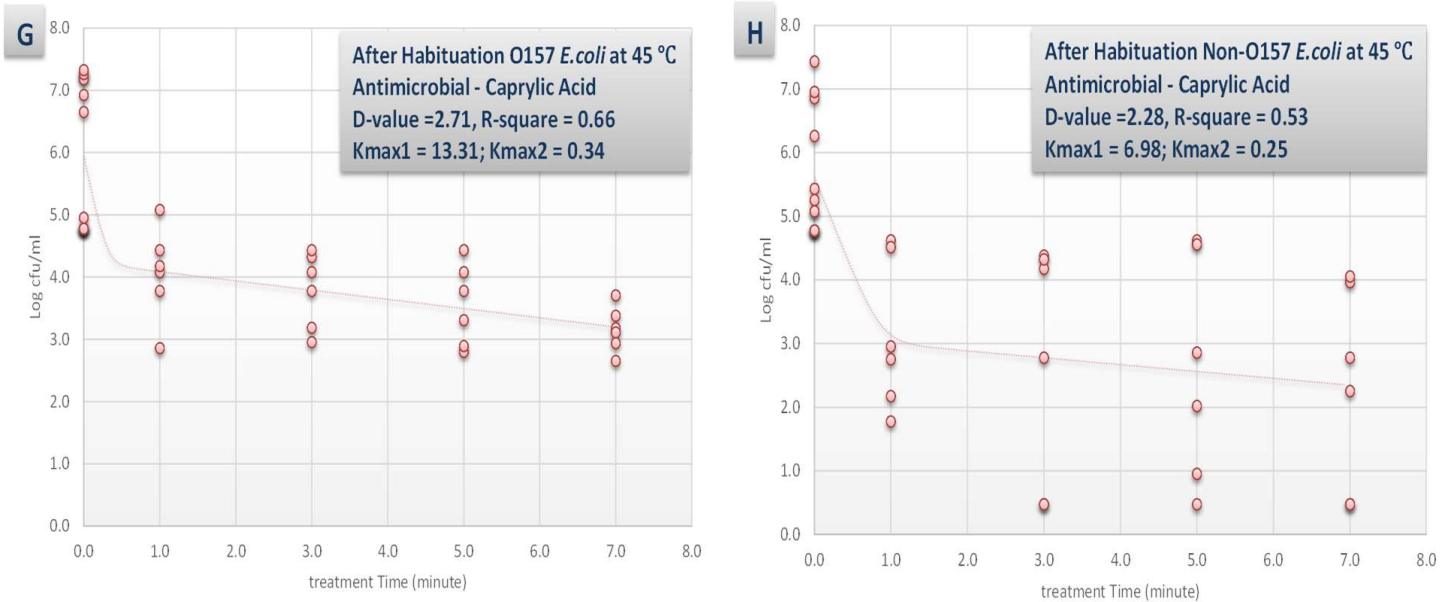

Figure 4. Inactivation rates for six-strain habituated and non-habituated mixture of E. coli O157:H7 (ATCC ${ }^{\circledR}$ numbers BAA 460, 43888, 43894, 35150, 43889, 43890) and the 'Big Six' non-O157 E. coli strain mixtures (ATCC ${ }^{\circledR}$ numbers BAA 2196, BAA 2193, BAA 2215, BAA 2440, BAA 2219, BAA 2192) exposed to $0.1 \%$ carvacrol, $0.1 \%$ caprylic acid, and elevated hydrostatic pressure at $450 \mathrm{MPa}$ (Barocycler Hub 440 , Pressure BioScience Inc., South Easton, MA) in sterilized blueberry juice. Using the GInaFiT software, the provided $K_{\max }$ values are selected from the best-fitted model (goodness-of-fit indicator of $\mathrm{R}^{2}$ values, $\alpha=0.05)$. $K_{\max }$ values indicate the expressions of number of $\log$ cycles of reduction in $1 / \mathrm{min}$ unit for each pressure/temperature combinations. Presented D-values are calculated based on best-fitted linear model, showing time required for one $\log (90 \%)$ of microbial cell reductions of the habituated microbial cell mixture. (A) Habituated E. coli $\mathrm{O} 157$ treated by no antimicrobial at $45^{\circ} \mathrm{C}$ with $\mathrm{R}^{2}=0.53$; (B) Habituated E. coli non-O157 treated by no antimicrobial at $45{ }^{\circ} \mathrm{C}$ with $\mathrm{R}^{2}=0.64$; (C) Non-habituated E. coli $\mathrm{O} 157$ treated by no antimicrobial at $45^{\circ} \mathrm{C}$ with $\mathrm{R}^{2}=0.48$; (D) Non-habituated $E$. coli non-O157 treated by no antimicrobial at $45^{\circ} \mathrm{C}$ with $\mathrm{R}^{2}=0.56$; (E) Habituated E. coli O157 treated by carvacrol $(0.1 \%)$ at $45^{\circ} \mathrm{C}$ with $\mathrm{R}^{2}=0.69 ;(\mathrm{F})$ Habituated E. coli non-O157 treated by carvacrol $(0.1 \%)$ at $45^{\circ} \mathrm{C}$ with $\mathrm{R}^{2}=0.78 ;(\mathrm{G})$ Habituated E. coli $\mathrm{O} 157$ treated by caprylic acid $(0.1 \%)$ at $45^{\circ} \mathrm{C}$ with $\mathrm{R}^{2}=0.81$; (H) Habituated E. coli non-O157 treated by caprylic acid $(0.1 \%)$ at $45^{\circ} \mathrm{C}$ with $\mathrm{R}^{2}=0.75$.

The synergistic effects of the tested antimicrobial ( $0.1 \%$ concentration) and habituation were less pronounced at elevated temperature of $45^{\circ} \mathrm{C}$ (Figure 4). For example, the D-values for habituated with no antimicrobial, non-habituated with no antimicrobial, habituated and treated with $0.1 \%$ carvacrol, and habituated and treated with $0.1 \%$ caprylic acid for STEC samples were similar $(p<0.05)$ and were 1.65, 1.51, 2.84, and $2.71 \mathrm{~min}$, respectively (Figure 4A,C,E,G). This indicates that additional of antimicrobials could appreciable enhance the decontamination efficacy of a pressure-based intervention at $4{ }^{\circ} \mathrm{C}$ while could have minor to no effects for augmenting the efficacy of a thermal-assisted high pressure pasteurization.

As further discussed in the introduction, antimicrobials used in the current study have Generally Recognized as Safe status in the United States regulatory landscape [28,45] and the concentrations utilized are similar to those used previously in literature [46]. As for any product development project, incorporation of these antimicrobials in a product formula for enhancing safety of the product, requires product specific and close attention to organoleptic properties of the product with and without the antimicrobials.

It is also noteworthy that this study utilized a six-strain mixture of E. coli O157:H7 and a six-strain mixture of non-O157 Shiga toxin-producing E. coli. As delineated in Section 2.1, these were selected based on our previously published screening trials as well as the strains' public health significance. Acid tolerance, sensitivity to intrinsic and extrinsic factors of a product, and reduction as a result of a thermal or non-thermal treatment could vary immensely among the plethora of Shiga toxin-producing isolates of the pathogen. Conducting experiments with similar design to the current study in future, using an array of individual strains followed by further analyses of the survivors after the treatments could be 
experiments of utmost importance and a complement to the current study for better assimilation of sensitivity of this pathogen of public health concern to pressure-based interventions under various intrinsic and extrinsic conditions of a product and processing conditions.

\section{Conclusions}

Under the condition of our experiments, for the vast majority of tested time and pressure intervals in presence or absence of two antimicrobials, O157 and non-O157 serogroups of Shiga toxin-producing Escherichia coli exhibited similar sensitivity to elevated hydrostatic pressure. Thus, if a pressure-based treatment is validated and is efficacious for decontamination of O157 serogroups of Escherichia coli, it would almost certainly exhibit comparable efficacy for reduction of non-O157 serogroups of the pathogen as well. We also observed that, particularly for treatments at $4{ }^{\circ} \mathrm{C}$, habituation of samples could meaningfully alter the results of a microbial challenge study and thus would need to be carefully considered for maximizing the external validity of a validation study. Reducing the cost of pressure-based treatments are currently the major curtailment for further adaption of this emerging technology. Our study indicates that application of natural antimicrobials could augment the decontamination efficacy of this technology, allowing the practitioners to benefit from synergism of natural antimicrobials and elevated hydrostatic pressure, to utilize lower intensity of the treatment with the same level of microbiological safety. This could be a practical solution for ultimately reducing high-pressure processing operation costs and increasing the competitiveness of products manufactured with this technology. This could also lead to enhanced preservation of nutritional and sensory properties of the products since mild hydrostatic pressure treatments are typically associated with no or minimal deleterious effects on physiochemical and organoleptic properties of food products.

Supplementary Materials: Supplementary materials can be found at http://www.mdpi.com/2076-2607/7/5/145/s1.

Author Contributions: M.N.K.: Conducted the main and preliminary trials, assisted in data management and analyses, co-wrote the first version of the manuscript. S.A.: Assisted in conduct of the trials, data collection, and data management. A.A.: Assisted in conduct of the trials, data collection, and data management. J.A.: Assisted in conduct of the trials, data collection, and data management. S.C.: Assisted in conduct of the trials, data collection, data management, and supervision of the students. A.F.: Secured extramural funding, designed the experiments and preliminary trials, prepared the inferential analyses code, supervised the students, co-wrote, revised and edited the manuscript.

Funding: Financial support in part from the National Institute of Food and Agriculture of the United States Department of Agriculture (Projects 2017-07534; 2017-07975; 2017-06088) and Pressure BioScience Inc. is acknowledged gratefully by the corresponding author.

Acknowledgments: Technical assistance and contributions of the members of the Public Health Microbiology Laboratory is sincerely appreciated by the authors. Authors also appreciate the feedback of anonymous reviewers and editorial team of Microorganisms.

Conflicts of Interest: The authors declare no conflict of interest. The funding sponsors have no role in design, data collection, microbiological and statistical analyses, or data interpretation and writing of the manuscript. The content of the current publication does not necessarily reflect views of the funding agencies.

\section{References}

1. United States Department of Agriculture, 2015-2020 Dietary Guidelines for Americans. Available online: https://health.gov/dietaryguidelines/2015/ (accessed on 1 May 2019).

2. Olaimat, A.N.; Holley, R.A. Factors influencing the microbial safety of fresh produce: A review. Food Microbiol. 2012, 32, 1-19. [CrossRef]

3. Warriner, K.; Huber, A.; Namvar, A.; Fan, W.; Dunfield, K. Recent advances in the microbial safety of fresh fruits and vegetables. Food Nutr. Res. 2009, 57, 155-208.

4. Fouladkhah, A. The Need for Evidence-Based Outreach in the Current Food Safety Regulatory Landscape. Commentary section. J. Ext. 2017, 55, 2 COM1.

5. Scallan, E.; Hoekstra, R.M.; Angulo, F.J.; Tauxe, R.V.; Widdowson, M.A.; Roy, S.L.; Jones, J.L.; Griffin, P.M. Foodborne illness acquired in the United States-Major pathogens. Emerg. Infect. Dis. 2011, 17, 7-15. [CrossRef] [PubMed] 
6. Crim, S.M.; Griffin, P.M.; Tauxe, R.; Marder, E.P.; Gilliss, D.; Cronquist, A.B.; Cartter, M.; Tobin-D'Angelo, M.; Blythe, D.; Smith, K.; et al. Preliminary incidence and trends of infection with pathogens transmitted commonly through food-Foodborne Diseases Active Surveillance Network, 10 US sites, 2006-2014. MMWR 2015, 64, 495. [PubMed]

7. Johnson, K.E.; Thorpe, C.M.; Sears, C.L. The emerging clinical importance of non-O157 Shiga toxin-producing Escherichia coli. Clin. Infect. Dis. 2006, 43, 1587-1595.

8. Bosilevac, J.M.; Arthur, T.M.; Bono, J.L.; Brichta-Harhay, D.M.; Kalchayanad, N.; King, D.A.; Shackelford, S.D.; Wheeler, M.L.; Koohmaraie, M. Prevalence and enumeration of Escherichia coli O157: H7 and Salmonella in U.S. abattoirs that process fewer than 1,000 head of cattle per day. J. Food Prot. 2009, 72, 1272-1278. [CrossRef]

9. Fouladkhah, A.; Geornaras, I.; Yang, H.; Sofos, J. Lactic Acid Resistance of Shiga Toxin-Producing Escherichia coli and Multidrug-resistant and Susceptible Salmonella Typhimurium and Salmonella Newport in Meat Homogenate. Food Microbiol. 2013, 36, 260-266. [CrossRef]

10. Brooks, J.T.; Sowers, E.G.; Wells, J.G.; Greene, K.D.; Griffin, P.M.; Hoekstra, R.M.; Strockbine, N.A. Non-O157 Shiga toxin-producing Escherichia coli infections in the United States, 1983-2002. J. Infect. Dis. 2005, 192, 1422-1429. [CrossRef]

11. Pihkala, N.; Bauer, N.; Eblen, D.; Evans, P.; Johnson, R.; Webb, J.; Williams, C.; FSIS. Risk Profile for Pathogenic Non-O157 Shiga Toxin-producing Escherichia coli. Available online: https://www.fsis.usda.gov/shared/PDF/ Non_O157_STEC_Risk_Profile_May2012.pdf (accessed on 22 May 2019).

12. Cutter, C.; Depasquale, D.; Hayes, J.; Raines, C.; Seniviranthne, R. Meat Science Review: HPP, Ground Beef and the 'Big 6' STEC. The National Provisioner. Available online: http://www.provisioneronline.com/articles/ 98113-meat-science-reviewehppeground-beef-and-theebig-6estec (accessed on 21 April 2013).

13. Centers for Disease Control and Prevention (CDC), National Outbreak Reporting System (NORS). 2018. Available online: https://wwwn.cdc.gov/norsdashboard/ (accessed on 1 May 2019).

14. Brown, M.H.; Booth, I.R. Acidulants and low pH. In Food Preservatives; Russell, N.J., Gould, G.W.Ž, Eds.; Springer: Berlin/Heidelberg, Germany, 1991; pp. 22-43.

15. O'Driscoll, B.; Gahan, C.G.M.; Hill, C. Adaptive acid tolerance response in Listeria monocytogenes: Isolation of an acid-tolerant mutant, which demonstrates, increased virulence. Appl. Environ. Microbiol. 1996, 62, 1693-1698.

16. Cheng, H.Y.; Chou, C.C. Survival of acid-adapted Escherichia coli O157: H7 in some acid foods and subsequent environmental stress. MS thesis, National Taiwan University, Taipei, Taiwan, 1999.

17. Foster, J.W.; Hall, H.K. Adaptive acidification tolerance response of Salmonella typhimurium. J. Bacteriol. 1990, 172, 771-778. [CrossRef]

18. Morgan, D.; Newman, C.P.; Hutchinson, D.N.; Walker, A.M.; Rowe, B.; Majid, F. Verotoxin producing Escherichia coli O157: H7 infections associated with the consumption of yoghurt. Epidemiol. Infect. 1993, 111, 181-187. [CrossRef]

19. Weagant, S.D.; Bryant, J.L.; Bark, D.H. Survival of Escherichia coli O157: H7 in mayonnaise-based sauces at room and refrigerated temperatures. J. Food Prot. 1994, 57, 629-631. [CrossRef]

20. Besser, R.E.; Lett, S.M.; Weber, J.T.; Doyle, M.P.; Barrett, T.J.; Wells, J.G.; Griffin, P.M. An outbreak of diarrhea and hemolytic uremic syndrome from Escherichia coli O157: H7 in fresh-pressed apple cider. J. Am. Med. Assoc. 1993, 269, 2217-2220. [CrossRef]

21. Cheng, H.Y.; Chou, C.C. Acid adaptation and temperature effect on the survival of Escherichia coli O157: H7 in acidic fruit juice and lactic fermented milk product. Int. J. Food Microbiol. 2001, 70, 189-195.

22. Luna, R.E.; Mody, R. Non-O157 Shiga Toxin-Producing E. coli (STEC) Outbreaks, United States. Available online: http://blogs.cdc.Gov/publichealthmatters/files/2010/05/nono157stec_obs_052110.pdf (accessed on 22 April 2013).

23. Allison, A.; Daniels, E.; Chowdhury, S.; Fouladkhah, A. Effects of elevated hydrostatic pressure against mesophilic background microflora and habituated Salmonella serovars in orange juice. Microorganisms 2018, 6, 23. [CrossRef]

24. Cao, X.M.; Zhang, Y.; Zhang, F.S.; Wang, Y.T.; Yi, J.Y.; Liao, X.J. Effects of high hydrostatic pressure on enzymes, phenolic compounds, anthocyanins, polymeric color and color of strawberry pulps. J. Sci. Food Agric. 2011, 91, 877-885. [CrossRef] [PubMed]

25. Han, Y.; Jiang, Y.; Xinglian, S.; Xinsheng, B.; Zhou, G. Effect of high pressure treatment on microbial populations of sliced vacuum-packed cooked ham. Meat Sci. 2011, 88, 682-688. [CrossRef] [PubMed] 
26. Hiremath, H.D.; Ramaswamy, H.S. High-pressure destruction kinetics of spoilage and pathogenic microorganisms in mango juice. J. Food Process. Preserv. 2012, 36, 113-125. [CrossRef]

27. Allison, A.; Chowdhury, S.; Fouladkhah, A. Synergism of Mild Heat and High-Pressure Pasteurization Against Listeria monocytogenes and Natural Microflora in Phosphate-Buffered Saline and Raw Milk. Microorganisms 2018, 6, 102. [CrossRef] [PubMed]

28. Vasudevan, P.; Marek, P.; Nair, M.K.M.; Annamalai, T.; Darre, M.; Khan, M.; Venkitanarayanan, K. In vitro inactivation of Salmonella Enteritidis in autoclaved chicken cecal contents by caprylic acid. J. Appl. Poult. Res. 2005, 14, 122-125. [CrossRef]

29. The U.S. Food and Drug Administration. 21CFR184.1025: Caprylic acid. Available online: https://www. accessdata.fda.gov/scripts/cdrh/cfdocs/cfcfr/CFRSearch.cfm?fr=184.1025 (accessed on 20 May 2019).

30. Annamalai, T.; Nair, M.K.M.; Marek, P.; Vasudevan, P.; Schreiber, D.; Knight, R.; Hoagland, T.; Venkitanarayanan, K. In vitro inactivation of Escherichia coli O157: H7 in bovine rumen fluid by caprylic acid. J. Food Prot. 2004, 67, 884-888. [CrossRef] [PubMed]

31. Nair, M.K.M.; Vasudevan, P.; Hoagland, T.; Venkitanarayanan, K. Inactivation of Escherichia coli O157: H7 and Listeria monocytogenes in milk by caprylic acid and monocaprylin. Food Microbiol. 2004, 21, 611-616. [CrossRef]

32. Chang., S.-S.; Redondo-Solano, M.; Thippareddi, H. Inactivation of Escherichia coli O157: H7 and Salmonella spp. on alfalfa seeds by caprylic acid and monocaprylin. Int. J. Food Microbiol. 2010, 144, 141-146. [CrossRef] [PubMed]

33. Burt, S. Essential oils: Their antibacterial properties and potential applications in foods-a review. Int. J. Food Microbiol. 2004, 94, 223-253. [CrossRef] [PubMed]

34. Ultee, A.; Slump, R.A.; Steging, G.; Smid, E.I. Antimicrobial activity carvacrol toward Bacillus cereus on rice. J. Food Prot. 2000, 63, 620-624. [CrossRef] [PubMed]

35. Lu, Y.; $\mathrm{Wu}, \mathrm{C}$. Reduction of Salmonella enterica contamination on grape tomatoes by washing with thyme oil, thymol, and carvacrol as compared with chlorine treatment. J. Food Prot. 2010, 73, 2270-2275. [CrossRef]

36. Allison, A.; Chowdhury, S.; Fouladkhah, A. Effects of Lactic Acid and Elevated Hydrostatic Pressure against Wild-Type and Rifampicin-Resistant O157 and Non-O157 Shiga Toxin-Producing Escherichia coli in Meat Homogenate. In Proceedings of the 2018 Annual Meeting of Institute of Food Technologists, Chicago, IL, USA. Available online: https://ift.planion.com/Web.User/AbstractDet?ACCOUNT=IFT\&ABSID=21564\& CONF=IFT18\&ssoOverride $=$ OFF\&CKEY $=($ accessed on 22 May 2019).

37. Fouladkhah, A.; Geornaras, I.; Sofos, J.N. Effects of Reheating against Listeria monocytogenes Inoculated on Cooked Chicken Breast Meat Stored Aerobically at $7{ }^{\circ} \mathrm{C}$. Food Prot. Trends 2012, 32, 697-704.

38. Koutsoumanis, K.P.; Sofos, J.N. Comparative acid stress response of Listeria monocytogenes, Escherichia coli O157: H7 and Salmonella Typhimurium after habituation at different $\mathrm{pH}$ conditions. Lett. Appl. Microbiol. 2004, 38, 321-326. [CrossRef]

39. Fouladkhah, A.; Geornaras, I.; Nychas, G.J.; Sofos, J.N. Antilisterial properties of marinades during refrigerated storage and microwave oven reheating against post-Cooking inoculated chicken breast meat. J. Food Sci. 2013, 78, M285-M289. [CrossRef]

40. Allison, A.; Fouladkhah, A. Sensitivity of Salmonella serovars and natural microflora to high-pressure pasteurization: Open access data for risk assessment and practitioners. Data Brief 2018, 21, 480-484. [CrossRef] [PubMed]

41. Geeraerd, A.H.; Valdramidis, V.P.; Van Impe, J.F. GInaFiT, a freeware tool to assess non-log-linear microbial survivor curves. Int. J. Food Microbiol. 2005, 102, 95-105. [CrossRef]

42. Ryu, J.H.; Beuchat, L.R. Influence of acid tolerance responses on survival, growth, and thermal cross-protection of Escherichia coli O157: H7 in acidified media and fruit juices. Int. J. Food Microbiol. 1988, 45, 185-193. [CrossRef]

43. Haberbeck, L.U.; Wang, X.; Michiels, C.; Devlieghere, F.; Uyttendaele, M.; Geeraerd, A.H. Cross-protection between controlled acid-adaptation and thermal inactivation for 48 Escherichia coli strains. Int. J. Food Microbiol. 2017, 241, 206-214. [CrossRef]

44. Fouladkhah, A.; Avens, J.S. Effects of combined heat and acetic acid on natural microflora reduction on cantaloupe melons. J. Food Prot. 2010, 73, 981-984. [CrossRef] [PubMed] 
45. The U.S. Food and Drug Administration, Code of Federal Regulation Title 21. Available online: https:// www.accessdata.fda.gov/scripts/cdrh/cfdocs/cfcfr/CFRSearch.cfm?CFRPart=172\&showFR=1 (accessed on 20 May 2019).

46. Moschonas, G.; Geornaras, I.; Stopforth, J.D.; Wach, D.; Woerner, D.R.; Belk, K.E.; Smith, G.C.; Sofos, J.N. Activity of caprylic acid, carvacrol, $\varepsilon$-polylysine and their combinations against Salmonella in not-ready-to-eat surface-browned, frozen, breaded chicken products. J. Food Sci. 2012, 77, M405-M411. [CrossRef]

(C) 2019 by the authors. Licensee MDPI, Basel, Switzerland. This article is an open access article distributed under the terms and conditions of the Creative Commons Attribution (CC BY) license (http://creativecommons.org/licenses/by/4.0/). 